\title{
Europäische Finanzkrise: Staatsverschuldung und Politikdefizite
}

\author{
FRANK GERLACH, WINFRIED HEIDEMANN
}

Zur Eindämmung der europäischen Finanzkrise haben Staats- und Regierungschefs, EU-Kommission und Europäische Zentralbank (EZB) in den letzten Monaten eine Reihe von Notmaßnahmen ergriffen. Im Dezember 2011 haben sie sich auf einen multilateralen Vertrag mit dem Ziel verständigt, die Währungs- um eine Fiskalunion mit bindenden Haushaltsvorschriften für die Mitgliedstaaten zu ergänzen. Über Konzeptionen und Vorschläge gab es heftige Auseinandersetzungen. Auf der einen Seite wird vertreten, primär die Akteure auf den Finanzmärkten seien Verursacher der Krise und ein zentrales Problem bestehe in den Ungleichgewichten im Intrahandel in der Eurozone, u. a. in den anhaltenden Leistungsbilanzüberschüssen Deutschlands. Eine entgegengesetzte Position stellt den jahrzehntelangen Aufwuchs staatlicher Schulden sowie die dauernde Verletzung der MaastrichtKriterien als entscheidende Anstöße für die aktuellen Probleme auf den Finanzmärkten heraus. Entsprechend variieren die Therapievorschläge. Während eine Seite vorrangig auf finanzielle Stützung der Defizitstaaten durch die EU und eine Stärkung der Binnennachfrage in Deutschland zum Abbau der Außenhandelsüberschüsse setzt, fokussiert eine andere auf Schuldenabbau und Konsolidierung der öffentlichen Haushalte in allen Staaten und das in den europäischen Verträgen verankerte Prinzip der Eigenverantwortung der Mitgliedstaaten für ihre Schulden. Wie sehr die Bewertungen divergieren, wird zudem an der Kommentierung der Beschlüsse vom Dezember 2011 deutlich: Neben der Bewertung, Europa sei der Lösung der Krise zumindest „einen Schritt näher" gekommen, ${ }^{\bullet}$ steht das harsche Urteil, es handele es sich um „intellektuellen Bankrott“.

Mit unserem Debattenbeitrag ${ }^{\boldsymbol{B}}$ wollen wir statt auf das Versagen der Finanzmärkte auf Politikdefizite und Staatsverschuldung als zentrale Krisenursachen aufmerksam machen und die Grenzen einer rein makroökonomischen Betrachtung ohne Berücksichtigung struktureller Zusammenhänge aufzeigen. Eine umfassende
Erklärung der Krisenerscheinungen können wir nicht geben.

\section{Politikmuster in den Krisenstaaten}

Am Beispiel Griechenlands lassen sich Politikdefizite als Ursachen der Probleme des Landes am deutlichsten erkennen. Griechenland war für die Aufnahme in die Euro-Zone nicht hinreichend wettbewerbsfähig, institutionell nicht vorbereitet und hat mit „Bilanzierungstricks“ oder riskanten Finanztransaktionen, wie z. B. der rechtswidrigen Verbriefung von EU-Mitteln, seine Haushaltsdaten manipuliert. Banken haben daran mitgewirkt und davon profitiert, die EU-Kommission und die Mitgliedstaaten haben es toleriert. Nach Einführung des Euro hat das Land die nun deutlich niedrigeren Zinsen für seine Staatsanleihen nicht zu einer Konsolidierung der Staatsfinanzen genutzt, sondern den Aufbau der Schulden fortgesetzt. In keinem Jahr hat das Land die Maastricht-Kriterien erfüllt. An den wirtschaftlichen Strukturen des Landes hat auch die heute vielfach geforderte „Marshall-Plan-Hilfe“ durch die EU-Strukturfonds in Höhe zweistelliger Milliardenbeträge nichts geändert. Eindeutig ist das Urteil von ExPremier Papandreou: „Wir haben über unsere Verhältnisse gelebt. Als Mitglied der Euro-Zone war es leicht für Griechenland, billige Kredite zu bekommen. Und dieses Geld haben wir falsch investiert. Wir haben damit ein System gefüttert, das nicht wettbewerbsfähig war. “ Dieses Politikversagen hat viele Ursachen in den Strukturen von Wirtschaft und Gesellschaft: Hier sind Klientilismus, Korruption, Ineffektivität des Staatsapparates u.a. bei der Steuererhebung, steuerliche Begünstigungen, Lohnverzerrungen zugunsten eines aufgeblähten öffentlichen Sektors und wachstumshemmende Regulierungen von Wirtschafts- und Berufszweigen durch Konzessionierungen zu nennen.

Italien hat ebenfalls mit Bilanzierungstricks und riskanten Finanztransaktionen seine Wirt- schaftsdaten vor Beitritt zur Euro-Zone manipuliert und anschließend die niedrigen Zinsen des Euro-Raums nicht für einen Schuldenabbau genutzt. ${ }^{\ominus}$ Es hat die $60 \%$-Grenze für den Gesamt-Schuldenstand ständig verletzt und lag auch bei der Neuverschuldung ab 2001 bis auf die Jahre 2007 und 2008 über der 3\%-Grenze. Trotz Verletzung der Maastricht-Kriterien gab es keine Konsequenzen. Jedoch ist das Land ökonomisch gesehen viel wettbewerbsfähiger als Griechenland. Es wäre allerdings zu kurz gegriffen, das „bad government" der Ära Berlusconi als allein verantwortlich anzuprangern. Vielmehr steht dahinter eine tief greifende Krise der Strukturen in Politik und Gesellschaft, die erst die Ära Berlusconi möglich machte. Deutliche Worte zum Versagen der Politik fand Ministerpräsident Monti: Der gigantische italienische Schuldenberg sei nicht die Schuld der Europäer, sondern der Italiener. Wenn die jungen Menschen in Italien heute keine Arbeit fänden, sei das die Folge einer Politik in Rom, die viele Jahre lang nur die eigenen Chancen bei den nächsten Wahlen im Auge hatte ${ }^{\bullet}$ - ein Urteil, das auch von vielen italienischen Gewerkschaftsvertretern geteilt wird.

Spanien ist ein anderer Fall. Das Land erfüllte lange Zeit die Maastricht-Kriterien. Jedoch

Fichtner, F. (2011) Nach der Schicksalswoche: Zu kurz gesprungen in: DIW Wochenbericht 78 (50), S.16.

(2) So G. Horn, zitiert nach Süddeutsche Zeitung vom 04.01.2012, S. 19.

(3) Für eine kritische Durchsicht des Manuskripts sind wir Lothar Kamp zu Dank verpflichtet.

$(4$ Zitiert nach Der Spiegel 2011 (48), S. 97.

5 Vgl. Erber, G. (2011): Italy's Fiscal Crisis, in: Intereconomics, Review of European Economic Policy 76 (6), S. 332-339.

6 Spiegel online vom 05.12.2011: Montis Sparplan: „Wir müssen die Welt retten“. 
beruhte seine positive Entwicklung wesentlich - begünstigt durch Kapitalimporte - auf dem Boom im Immobiliensektor, der für die Wirtschaftsstruktur immer wichtiger wurde und sich mehr und mehr zu einer Blase entwickelte. Obwohl auf diese Gefahren hingewiesen, reagierte die spanische Regierung nicht mit adäquaten Maßnahmen.

In Irland war es der Zusammenbruch des von der Regierung geförderten hochspekulativen privaten Finanzsektors sowie ebenfalls eine "Immobilienblase“, in Portugal die sich - trotz EU-Strukturhilfen - sukzessiv verschlechternde Wettbewerbsfähigkeit, die zu einem massiven Anstieg der Schulden und dann für Portugal ab 2004, für Irland ab 2008 zu einer Verletzung der Maastricht-Kriterien führte.

Die Ursachen für die Krise, die hier nur angerissen werden können, sind länderspezifisch zu analysieren. In allen Fällen ist jedoch deutlich, dass die Lösung der Schuldenprobleme zugunsten jeweils aktueller Interessenkonstellationen weit in die Zukunft verlagert wird. ${ }^{\mathbf{8}}$ Die Basis der Vertrauenskrise der Finanzmärkte wurde vor der Finanzkrise 2007/08 gelegt. Als der Zustand der griechischen und italienischen wie auch der spanischen, portugiesischen und infolge der Finanzkrise 2007 irischen Staatsfinanzen nicht zuletzt durch neue Transparenzvorschriften der EU offenkundig wurde, verlangten die Investoren auf den Finanzmärkten höhere Risikoaufschläge. Diese Entwicklung führte dazu, dass sich diese Länder nur noch mit hohen Kosten am Kapitalmarkt refinanzieren können. Die Maßnahmen in der Finanzmarktkrise 2007/08 führten zu einem weiteren Schuldenanstieg. Die Finanzmärkte wa ren aber keineswegs alleinige Verursacher - und deshalb greift die Schuldzuweisung an „Spekulanten“" zu kurz.

Rückblickend wird ein entscheidender „Webfehler" der Währungsunion deutlich: Ihre Einführung wurde nicht verbunden mit adäquaten fiskal- und wirtschaftspolitischen Kompetenzen der EU. Die „Offene Methode der Koordinierung“ erwies sich als zu schwach, um die divergierenden Wirtschafts- und Fiskalpolitiken der souveränen Mitgliedstaaten zusammenzubinden. Die Maastricht-Kriterien für Defizitgrenzen wurden ignoriert, die vorgesehenen Sanktionen nicht beschlossen. Insbesondere die Politik Deutschlands und Frankreichs hat mit der Verletzung dieser Kriterien die Aufweichung des Vertrags begünstigt.

\section{Zur Problematik der Staatsschulden}

Keynesianische Konzepte rechtfertigen öffentliche Verschuldung zur Konjunkturankurbelung mit der Hoffnung auf anhaltendes Wachstum. Tatsächlich handelt es sich in der politischen Praxis dabei meist um einen „halbierten“ Keynes: In guten wirtschaftlichen Zeiten wurden bislang in aller Regel weder Konjunkturrücklagen gebildet noch Schulden abgebaut, um Spielraum für Maß nahmen in der Krise zu haben, allenfalls wurde die Nettokreditaufnahme reduziert. Dabei ist der kontinuierliche Aufbau von Verschuldung in nahezu allen Staaten auf politische Prozesse zurückzuführen: Erwartungen in der Gesellschaft auf steigende Einkommen und soziale Sicherheit wurden von den Regierungen in nicht unbeträchtlichem Ausmaß durch Verschuldung befriedigt. $^{\bullet}$

Damit wurde die Brisanz steigender Schulden, wenn die Staaten sich für ihre Refinanzierung von den Finanzmärkten und damit auch deren Bewertungsmethoden abhängig machen, systematisch unterschätzt. In keynesianischen Betrachtungen scheint die Höhe der staatlichen Schulden für die Regierung nur eine nachgelagerte Rolle zu spielen. Im Sinne der Globalsteuerung sei vielmehr die Höhe der Nachfrage die zentrale Größe der Wirtschaftspolitik. Hohe Zinslasten des Staates würden der Gesellschaft insgesamt keine Last aufbürden, weil ihnen in gleicher Höhe zusätzliche Zinseinnahmen im privaten Sektor gegenüberstünden. Das ist nur im buchhalterischen Sinne richtig, wirtschaftsund sozialpolitisch aber höchst problematisch: Staatsverschuldung anstelle von sozial ausgewogenen Steuersystemen bringt den zumeist reicheren Gläubigern Zinsen und sorgt so für eine Umverteilung von unten nach oben; Staatspapiere werden nicht nur von Einheimischen gehalten, sondern auch von Ausländern - Zinsen fließen also auch ins Ausland; wenn höhere Steuern wegen höherer Staatsschulden erhoben werden, ist mit Ausweichreaktionen (z. B. Steuerflucht, Steuerhinterziehung) zu rechnen. ${ }^{\circledR}$ Ökonometrische Untersuchungen zeigen zudem, dass sich Staatsschulden (in Relation zum Bruttoinlandsprodukt) ab einem Niveau von ca. 80 bis $90 \%$ negativ auf das Wachstum auswirken. Über den genauen Schwellenwert lässt sich streiten, aber die Befunde dürfen nicht ignoriert werden. ${ }^{(1}$

\section{Außenwirtschaftliche Ungleichgewich- te als Krisenursache?}

Hinsichtlich der Ursachen der Krise ist umstritten, ob die wirtschaftlichen Ungleichgewichte in der Eurozone die zentrale Ursache für die Verwerfungen sind. In diesem Zusammenhang wird behauptet, das deutsche Wirtschafts- und Sozialmodell habe erheblich zur Instabilität in der Euro-Zone beigetragen. Durch eine exzessive Exportstrategie, die durch stagnierende Reallöhne gestützt wurde, seien extreme Ungleichgewichte im Außenhandel entstanden und die Binnennachfrage sei nicht vorangekommen. ${ }^{\text {(2) }}$ Nun hat eine detaillierte Untersuchung von $\mathrm{Ge}$ org Erber ${ }^{\text {(3) }}$ die Verantwortung Deutschlands für die Ungleichgewichte in der EU und der EuroZone relativiert. Nach den vorliegenden Daten war wegen der Wachstumsschwäche der EuroLänder die Expansion des Handels Deutschlands in Länder außerhalb der Euro-Zone, insbesondere in die BRIC-Staaten, ${ }^{\circledR}$ entscheidender. Die Euro-Zone hat von der Exportstärke Deutsch-
0 Issing, O. (2011): Interview mit CNN vom 01.05.2011. Danach haben die EZB und die Banco de Espagna die spanische Regierung frühzeitig gewarnt.

8 Vgl. Konrad, K. A./Zschäpitz, H. (2010): Schulden ohne Sühne, München, S. 86.

(9 Vgl. Streeck, W. (2011): The crisis in context, MPIfG Discussion Paper (11/15), Köln.

(10 Vgl. Konrad, K. A./Zschäpitz, H., a.a.O., S. 131ff.
(1) Vgl. Cechetti, S. G./Mohanty, M. S./Zampolli, F. (2011): The real effects of debt. BIS Working Paper (352), Social Science Research Network: http://ssrn.com/abstract=1946170; Furceri, D./Zdzienicka, A. (2011): How costly are debt crises? IMF Working Paper 11/280; Reinhart, C. M./Rogoff, K. S. (2010): Growth in a Time of Debt, NBER Working Papers (15639), Cambridge, MA.

(12) Vgl. Lehndorff, S. (2011): Deutschland in der europäischen Krise:Teil der Lösung oderTeil des Problems? in: WSI-Mitteilungen, 64 (12), S. 650-658, http://www.boeckler.de/cps/rde/ xchg/hbs/hs.xsl/wsi 38400 38420.htm; Niechoj, T./Stein, U./Stephan, S./Zwiener, R. (2011): Deutsche Arbeitskosten: Eine Quelle der Instabilität im Euroraum, IMK Report (68), Düsseldorf.

(13 Erber, G. (2012): Dichtung und Wahrheit Deutschlands Position bei Lohnstückkosten, Extra-Handel und realen effektiven Wechselkursen -Was sagt die Statistik? Social Science Research Network, http://ssrn.com/abstract=1978707.

(4) Zu den BRIC-Saaten zählen Brasilien, Russland, Indien, China. 
lands insofern profitiert, als sie sonst wohl ein sehr hohes Leistungsbilanzdefizit aufweisen würde. Inwieweit eine Stärkung der deutschen Binnennachfrage - jenseits des Tourismus - der Exportwirtschaft der Krisenstaaten aufhelfen könnte, wäre länderspezifisch genauer zu diskutieren. Strukturpolitisch (Dienstleistungen) und verteilungspolitisch (Niedriglohnsektor) wäre sie jedoch für die Bundesrepublik sinnvoll und wünschenswert. Uns scheint das Kernproblem, also die Verschlechterung der Wettbewerbsfähigkeit vieler Länder in der Eurozone, jedoch auf Defizite in den politischen und ökonomischen Strukturen zurückzuführen zu sein, die sich insbesondere in einer mangelnden Innovationsfähigkeit dokumentieren. Die Betrachtung allein makroökonomischer Aggregatgrößen - hier die Lohnstückkostenentwicklung - kann zu Fehlschlüssen führen. Zumindest sollte der Indikator „sektorale Lohnstückkosten“ hinzugezogen werden. Ein genauer und differenzierter Blick auf die jeweiligen Strukturen in den Staaten u. a. mit der Verwendung einer Vielzahl von Indikatoren ${ }^{\left({ }^{\circ}\right.}$ ist jedoch in jedem Falle erforderlich. Nicht zufällig legt die moderne Außenhandelstheorie einen starken Akzent auf die Innovationsfähigkeit. ${ }^{\circledR}$ Warum einige Volkswirtschaften langfristig im Vergleich zu anderen strukturell ins Hintertreffen geraten, beantworten keynesianische Denkansätze nicht. Hierfür ist eine Analyse der längerfristigen spezifischen Akkumulationsbedingungen erforderlich, die bekanntlich Gegenstand der Wachstumstheorie und -politik sind. Die mangelnde Wettbewerbsfähigkeit Griechenlands und Portugals, ihre Schwächung in Italien, die fehlende industrielle Basis in Spanien (außer Katalonien und dem Baskenland) haben tiefe historische Wurzeln. Eine hohe Innovationsfähigkeit einer Volkswirtschaft, die Nutzung des „,first mover advantage“ im Schumpeterschen Sinne, erfordert ein adäquates Gerüst von Institutionen. Der an der Analyse von Institutionen orientierte Ansatz zielt genau darauf. ${ }^{\circledR}$ Ein Punkt ist uns in diesem Zusammenhang besonders wichtig: Empirische Untersuchungen der Hans-Böckler-Stiftung belegen die positive Rolle der Mitbestimmung für betriebliche Innovationsprozesse. ${ }^{\circledR}$ Es gibt gute Argumente dafür, dass die Wettbewerbsfähigkeit der deutschen Wirtschaft wichtige Ursachen im Spezifikum der industriellen Beziehungen hat: Mitbestimmung und Tarifpolitik haben Produktivitätsentwicklung gefördert und Beschäftigung gesichert. Damit haben sie entscheidenden Anteil am Entwicklungspfad der deutschen Wirtschaft wie auch an der schnellen Bewältigung der Folgen der Krise nach 2008.

\section{Geldpolitische Notlösungen}

Da die grundlegenden Vorschläge zur Lösung der Finanzkrise größere und von den Unwägbarkeiten nationaler politischer Willensbildungsprozesse abhängige Veränderungen des europäischen Vertragswerks voraussetzen, wurden seit 2010 nach und nach verschiedene Notmaßnahmen ergriffen. Im Kern führen bereits der Rettungsschirm, der Europäische Rettungsfonds, der Aufkauf von Staatsanleihen durch die EZB sowie die exorbitante Ausweitung der Target-II-Kreditlinien zur Finanzierung von Nettoverbindlichkeiten im europäischen Zahlungsverkehr in Richtung einer Vergemeinschaftung der Schulden. Dass die EZB dabei geldpolitisch die Grenzen ihre Aufgabenstellung verletzte, ist auch Folge der Defizite europäischer Politik, die keine Lösungsinstrumente für zahlungsunfähige Staaten entwickelt hatte. Eine beruhigende Wirkung dieser Maßnahmen auf die Finanzmärkte war nur temporär zu verzeichnen, weil diesen das Vertrauen in die langfristige Kapazität der Staaten, ihre Schuldenprobleme zu lösen, fehlte.

Vor diesem Hintergrund wurden geldpolitische Maßnahmen vorgeschlagen, die die „Feuerkraft“ der Krisenabwehr („bazooka“) verstärken sollten, de facto aber auf eine weitere Vergemeinschaftung der Schulden bei nur geringen Möglichkeiten der Beeinflussung von nationaler Wirtschafts- und Haushaltspolitik hinauslaufen:

(1) Gemeinsame Anleihen aller Mitgliedstaaten (Eurobonds). Die Bonität der ökonomisch starken Staaten soll Krisenstaaten niedrige Zinssätze garantieren und ihnen so eine leichtere Refinanzierung ermöglichen. Eurobonds sind aber hinsichtlich der Auswirkungen auf das Zinsniveau keineswegs harmlos. Ihre Befürworter vernachlässigen das Risiko von Zinsaufschlägen auch für die „starken“ Länder. Überdies wären sie derzeit europarechtlich (und in Deutschland möglicherweise auch verfassungsrechtlich) unzulässig, könnten also die aktuelle Krise ohnehin nicht lösen.

(2) Eine unbeschränkte Garantie der nationalen Staatsanleihen durch die Europäische Zentralbank. Die EZB hat bereits Anfang 2012 einen Bestand von über 200 Mrd. € im Rahmen von „Notmaßnahmen“ angekaufter Staatsanleihen. Die Verfechter dieses Vorschlages wollen jedoch darüber deutlich hinausgehen. Nach ihrer Ansicht kann die EZB die Ankäufe von Staatsanleihen durch Entzug von Liquidität an anderer Stelle „sterilisieren“. Ob dies unbegrenzt möglich ist, ist umstritten. Einen großen Teil der „sterilisierten“ Mittel können Banken bei der EZB abrufen und in inflationsfördernde Kredite umwandeln. Eine Garantie aller Staatsschulden durch die EZB würde in jedem Falle den Anreiz für die Konsolidierung der nationalen Haushalte schwächen (das Problem des „moral hazard“). Auch widerspräche dies dem Verbot der Finanzierung nationaler Haushalte durch die EZB.

Die ökonomische und rechtliche Problematik solcher Vorschläge führte zur Konstruktion des ständigen europäischen Rettungsfonds ESM. Um dessen Wirkung trotz begrenzter Mittel zu vergrößern, soll er durch zusätzliche Mittel privater wie auch staatlicher Investoren des Weltmarktes "gehebelt" werden. Bisher ist aber die Bereitschaft der Investoren zur Beteiligung recht gering.

\section{Vorbild FED?}

Häufig wird von den Befürwortern eines Ankaufs von Staatsanleihen auf die Rolle der amerikanischen Zentralbank FED verwiesen. Der grundlegende Unterschied besteht jedoch in der Vielzahl von fiskalpolitisch souveränen Staaten in Europa. Auch war die Ausrichtung der „angelsächsischen“ Politik auf Finanzierung von Staatsdefiziten durch die Zentralbank (Niedrigzinspolitik und „Fluten“ der Finanzmärkte) bisher abhängig von dem Vertrauen der Gläubigerstaaten, das jedoch nicht unbegrenzt garantiert ist. Die grundlegenden Probleme der USA (wie auch etlicher Staaten der EU-Zone), nämlich die mangelnde Wettbewerbsfähigkeit ihrer Industrien, werden durch das Fluten der Märkte

(15 Vgl. exemplarisch: Schwab, K./World Economic Forum (Hrsg.) (2011): The Global Competitiveness Report 2011-2012, Geneva

(16) Vgl. Eaton, J./Kortum, S. (2002): Technology, Geography and Trade, in: Econometrica 70 (5), S. 1741.

$(1)$ Vgl. z. B. Hall, P. A./Soskice, D. (2001): Varieties of Capitalism, The Institutional Foundations of Comparative Advantage, Oxford.

$(8$ Z. B. Haipeter, T./Brettschneider, A./Bromberg, T./ Lehndorff, S. (2011): Rückenwind für Betriebsräte - eine Analyse betrieblicher Modernisierungskampagnen in der Metall- und Elektroindustrie, Berlin; Kriegesmann, B./ Kley, T./Kublik, S./ (2010): Innovationstreiber betriebliche Mitbestimmung?, in: WSI-Mitteilungen 63 (2), S. 71-78, http://www.boeckler.de/cps/rde/xchg/ hbs/hs.xsl/wsi_25369_25378.htm 
mit immer mehr Geld keineswegs beseitigt. Die daraus resultierende Gefahr von inflationären Tendenzen geht nicht nur zulasten der Realwirtschaft, sondern immer auch der Ersparnisse der "kleinen Leute“. Die beiden amerikanischen Ökonominnen Carmen Reinhart und Belen Sbrancia haben nachgewiesen, dass die Lösung in vielen Schuldenkrisen in der Vergangenheit u. a. in der Inflationierung und damit der Enteignung der Sparer lag. ${ }^{(0)}$ Inzwischen sind in den USA die Staatsfinanzen zerrüttet. Die Sparquote lag seit Jahren bei null, die USA sind abhängig vom Kapitalimport aus aufstrebenden Schwellenländern wie insbesondere China.

\section{Ein erweiterter Blick}

Welche Lösungen sind möglich? Hilfreich kann ein Blick in die Ergebnisse wirtschaftshistorischer und soziologischer Forschung sein. Eine historische Betrachtung der Wirtschaftskrisen und ihrer Bewältigung in den letzten 150 Jahren zeigt, dass aus Analyse und Kritik heraus jeweils neue Problemlösungen entwickelt wurden. ${ }^{20}$ Der Historiker Jürgen Kocka schließt daraus, dass aktuelle Krisen nicht ohne Weiteres mit den Lösungsstrategien vorhergehender Krisen bewältigt werden können. Der langfristige Wandel vom Spar- über den Konsum- zum Pumpkapitalismus (Dahrendorf) ${ }^{2}$ hat die Steuerungsbedingungen der Wirtschaft so grundlegend verändert, dass die oben als „halbiert keynesianisch“ bezeichneten Rezepte die Probleme nur verschärfen können. Im letzten Jahr vor der Weltwirtschaftskrise 1928/29 lag die Verschuldung Deutschlands bei ca. $10 \%$, im letzten Jahr vor der Finanzkrise 2008/09 bei $62 \%$ des Bruttosozialprodukts. Angesichts dieser Zahlen folgert Kocka: „Wenn er [Keynes] heute lebte, würde er wahrscheinlich mehr darüber schreiben, was man tun kann, um den machtvollen Ausgabe-Trend einzudämmen und manchmal weniger Geld auszugeben als man verdient. Und er würde sich vielleicht mehr Sorgen um die längerfristige Zukunft machen." Bei dem von Dahrendorf beschriebenen historischen Wandel handelt es sich um einen Mentalitätswandel, in dessen Verlauf sich die
Einstellungen zu Sparen und Bedürfnisaufschub sowie zu Ausgeben und Konsum in allen Gesellschaftsschichten verändert haben. Dadurch wird die systemimmanente Spannung zwischen den Imperativen des Sparens und des Ausgebens oder zwischen Investition und Konsum auf eine neue Stufe der Instabilität gehoben (Kocka). Lösungen hierfür können jedenfalls nicht umstandslos in den alten makroökonomischen Formeln der Forcierung von Konsum und staatlichen Schulden gesucht werden. Im Übrigen sollte man in diesem Zusammenhang die Marx'sche Kritik an der Unterkonsumtionstheorie nicht zur Gänze vergessen.

Wichtig scheint uns zunächst die mittelund langfristige Rückführung der Staatsschulden. Dabei sollten im Sinne von Verteilungsgerechtigkeit „starke Schultern“ mehr tragen als „schwache“. Eine angesichts der Krise in manchen Staaten möglicherweise unabwendbare kurzfristig expansive Politik zur Wachstumsförderung kann nur im Rahmen politischer, rechtlicher, ökonomischer und sozialer Strukturreformen und mit bindenden Vorgaben des Schuldenabbaus nachhaltig sein. Wenn es keinen Rückfall in nationalstaatliche Problemlösungen geben soll und Binnenmarkt und Eurozone auf Dauer erhalten werden sollen, ist die Entwicklung zu „mehr Europa“ mit Stärkung seiner wirtschafts-, finanz- und auch sozialpolitischen Kompetenzen unausweichlich. Das muss aber mit einem Schub an demokratischer Legitimation verbunden werden, die bisher sowohl im Verhältnis zwischen den europäischen Institutionen als auch zwischen den Nationalstaaten und Europa unterentwickelt und labil ist. ${ }^{3}$ Dafür sind tief greifende Änderungen der europäischen Verträge nötig, wie sie nach den Beschlüssen der Staats- und Regierungschefs vom Dezember 2011 zunächst nur für die Wirtschafts- und Finanzpolitik auf der Tagesordnung stehen. Für Deutschland ist das wiederum eine Gratwanderung zwischen europäischen Ansprüchen und innerstaatlichen verfassungsrechtlichen Möglichkeiten. Eine wichtige Lehre aus der Krise der letzten drei Jahre wäre die Entwicklung einer neuen europa- weiten Stabilitätskultur. Dazu gehört nicht nur die stärkere Regulierung der Finanzmärkte und Finanzierungsinstrumente, um Marktversagen zu minimieren, sondern auch eine stringente Regulierung der nationalen Fiskalpolitiken mit verbindlichen Schuldenbremsen, automatischen Sanktionsmöglichkeiten und europäischen Klagerechten zur Minimierung von Staatsversagen. Das könnte den in politischen Prozessen angelegten Anreizen zur Schuldenausweitung entgegenwirken. Unabdingbar ist dafür auch die Achtung einer Kultur der Rechtsstaatlichkeit, die nicht immer wieder durch Vorschläge zur Umgehung von Vorschriften des europäischen Vertragswerks unterminiert werden darf.

(19) Reinhart, C. M./Sbrancia, M. B. (2011): The liquidation of government debt, NBER Working Paper (16893) Cambridge, MA.

(20) Kocka, J. (2011): Der Kapitalismus und seine Krisen in historischer Perspektive, in: Ders. (Hrsg.): Arbeiten an der Geschichte, Göttingen, S. 307-322.

21) Dahrendorf, R. (2009): Nach der Krise: Zurück zur protestantischen Ethik?, in: Merkur (720), S. $373-381$.

22 Kocka, a.a.O., S. $320 f$.

23 Siehe dazu: Habermas, J. (2011): Zur Verfassung Europas, Berlin.

\section{AUTOREN}

FRANK GERLACH, Dr., war bis 2009 Leiter des Referats "Strukturwandel - Innovation und Beschäftigung" in der Abteilung Forschungsförderung der Hans-Böckler-Stiftung

WINFRIED HEIDEMANN, Dr., ist Leiter des Referats "Qualifikation" in der Abteilung Mitbestimmungsförderung der Hans-Böckler-Stiftung.

winfried-heidemann@boeckler.de 